\title{
Cell-Specific Effects of Ethanol on Nitric Oxide Synthase Induced by Inflammatory Mediators
}

\author{
Patience 0. Obih ${ }^{*}$, Barry J. Potter ${ }^{2}$ \\ ${ }^{1}$ College of Pharmacy, Xavier University of Louisiana, New Orleans, LA, USA \\ ${ }^{2}$ Department of Physiology, Louisiana State University Health Sciences Center, New Orleans, LA, USA \\ Email: ${ }^{*}$ poobih@xula.edu
}

Received 13 October 2014; revised 29 November 2014; accepted 25 December 2014

Copyright (C) 2014 by authors and Scientific Research Publishing Inc.

This work is licensed under the Creative Commons Attribution International License (CC BY). http://creativecommons.org/licenses/by/4.0/

(c) (i) Open Access

\begin{abstract}
To examine the pro- and anti-oxidant properties of nitric oxide (NO) production in alcoholic liver disease, we compared the effects of ethanol pretreatment $(24 \mathrm{hrs}, 100 \mathrm{mM}$ concentration in a dedicated $\mathrm{CO}_{2}$ incubator) on the induction of inducible NO synthase and its activity in a cell culture system. We employed two types of liver cells as models for the intact liver parenchyma: the rat hepatoma cell FTO2B and rat hepatocytes in primary culture. Cells were incubated with a combination of cytokines (IFN- $\gamma$, TNF- $\alpha$, IL- $\beta$ ) and LPS in the presence or absence of (85 - $93 \mathrm{mM}$ ) ethanol in the culture media. At series of time intervals, production of nitric oxide was measured as the accumulation of nitrite and nitrate, using Griess assay. The results revealed that 1) total NO formation was attenuated by ethanol in hepatocytes (ca. 50\%) but augmented in FTO2B cells (ca. $37 \%$ ) and 2) both pretreatment and co-treatment with ethanol were necessary for maximal ethanol effect. These results indicate that the effects of ethanol on inflammatory processes, such as induction of NO synthesis, are cell-type specific.
\end{abstract}

\section{Keywords}

Ethanol, Nitric Oxide, FTO2B Cells, Hepatocytes, Tissue Culture

\section{Introduction}

Excessive alcohol use is associated with significant increase in short-term risks to health and safety, and the risk increases as the amount of drinking increases [1]. Center for Disease Control (CDC) also estimated that $17 \%$ of

\footnotetext{
*Corresponding author.
} 
men and $8 \%$ of women would be addicted to alcohol at some point in their life. Excessive use of alcohol increases the risk of cancer of mouth, esophagus, throat, liver, and colon in men. It also increases the incidence of infertility and delay in the development of secondary characters in men by interfering with male sex hormones. Alcohol is also the 3rd leading life-style related cause of death in the United States [1]. Long term use of alcohol has been known to cause illness and death from the liver disease [2]-[4]. The liver plays important roles in the metabolic and immune responses during sepsis [5] [6]. Both acute and chronic ethanol has been shown to be capable of causing immunomodulation. One important component of the immune response is the production of small reactive oxygen species, such as superoxide $\left(\mathrm{O}_{2}\right)$, hydrogen peroxide $\left(\mathrm{H}_{2} \mathrm{O}_{2}\right)$, and nitric oxide (NO) [7]. While the partially reduced oxygen species $\mathrm{O}_{2}$ and $\mathrm{H}_{2} \mathrm{O}_{2}$ possess pro-oxidant properties, NO can have either proor anti-oxidant actions [8]. The factors which determine whether NO in a particular setting is pro- versus anti-oxidant are largely unknown, but it is known that NO can exert powerful effects in vivo under conditions of oxidative stress. In addition, many of the cellular actions of ethanol may be as a result of oxidative stress. Chronic ingestion of alcohol generates free radicals that are injurious to the body. Alcohol promotes the generation of reactive oxygen species (ROS) through many processes and these are injurious to different cells in the body [9] [10]. Both acute and chronic exposure to alcohol can lead to peroxidation of proteins, lipids and DNA. Through various pathways alcohol can lead to development of alcoholic liver disease and damage to many other tissues in the body. Increasing oxidative stress by alcohol can activate the c-Jun N-terminal kinase/mitogen-activated protein kinase (JNK/MAP) signaling pathway, and JNK might be a target for the prevention of alcohol-induced steatosis [10]. Alcohol affects the immune system by altering the production of signaling cytokines which in turn activates an array of biochemical processes. Alcohol stimulates cytochrome P450 2E1 (CYP2E1) which metabolizes alcohol and many other substances to produce more ROS [11]. ROS are toxic to cells because they can react with macromolecules like proteins, lipids, and DNA. Chronic alcohol use has been shown to increase iron absorption from food [10]. It has been shown that adding iron to alcohol containing diet increases liver injury in animals while adding agents that capture the free radicals can prevent or ameliorate the toxic effects of alcohol on the liver [11] [12]. Nitric oxide is a major immune effector with both pro-inflammatory and anti-inflammatory actions. It is produced by a variety of cell types, plays diverse roles as an immunomodulator, neurotransmitter, and vasodilator. NO can take part in a variety of biologically important reactions, which are mediated through oxygen species and transition metals. The cellular targets of NO reaction include iron-containing proteins and complexes, low and high molecular weight thiols, amine-containing targets such as nucleic acids, phenolic groups such as tyrosine, and cellular radicals. NO plays many different roles in depending on the conditions and levels of its production. Abnormal NO production has been linked to a number of clinically important conditions. In this study we have compared the effect of ethanol in nitric oxide production in two cell types, rat primary hepatocytes and rat hepatoma cell line, FTO2B.

\section{Materials and Methods}

\subsection{Isolation, Cell Culture, and Treatment of Hepatocytes}

Hepatocytes were isolated from male Sprague Dawley rats (175 - 250 g) using a modification of in situ collagenase perfusion technique [13]. Immediate viability was assessed using trypan blue and long term viability from LDH leakage into the medium [12]. The cells thus obtained were purified further by percoll gradient centrifugation to remove nonparenchymal cells. The cells were cultured overnight at $37^{\circ} \mathrm{C}$ in an atmosphere of $5 \% \mathrm{CO}_{2}$ 95\% air in Williams E medium containing $1 \mathrm{U} / \mathrm{ml}$ insulin, $2 \mathrm{mM}$ glutamine, $10^{5} \mathrm{UI}$ penicillin, $100 \mathrm{mg} / \mathrm{l} \mathrm{strep-}$ tomycin, and $10 \%$ low endotoxin fetal calf serum in 12-well plates at a density of 200,000 cells per well. The cultures were incubated in Williams medium $\mathrm{E}$ (with serum) with additions indicated in the text.

\subsection{FT02B Cells}

The rat hepatoma cell line FTO2B was grown at $37^{\circ} \mathrm{C}$ in a $5 \% \mathrm{CO}_{2}$ atmosphere in Dulbecco's modified Eagle's medium/Ham's F12 medium (1:1) supplemented with 10\% nonheat-inactivated bovine calf serum, $2 \mathrm{mM}$ glutamine, $50 \mathrm{U} / \mathrm{ml}$ penicillin-streptomycin, and $0.2 \%$ sodium bicarbonate. FTO2B cells $\left(2 \times 10^{5} / \mathrm{ml}\right)$ were plated in 12-well plates and allowed to adhere overnight before an experiment. Cells were used for experiments only when they were $90 \%$ confluent or more. 


\subsection{Treatment}

The effect of ethanol pretreatment (100 mM concentration for 24 hrs in a dedicated $\mathrm{CO}_{2}$ incubator) on the induction of inducible NO synthase and its activity were studied on two types of liver cells, namely: the rat hepatoma cell FTO2B and primary rat hepatocytes. The cells were incubated with a combination of inflammatory mediators to induce NO synthase: IFN- $\gamma$, TNF- $\alpha$, IL- $\beta$, and LPS. This combination has previously been shown to induce maximal expression and activity of iNOS in isolated rat and human hepatocytes, and the amounts of NO produced by human hepatocytes are the highest for any human cell reported. The amounts of the inflammatory mediators used were as follows: Rat IFN- $\gamma(100 \mathrm{U} / \mathrm{ml})$, TNF- $\alpha(500 \mathrm{U} / \mathrm{ml}), \mathrm{IL}-\beta(100 \mathrm{U} / \mathrm{ml}), \mathrm{LPS}(10 \mu \mathrm{g} / \mathrm{ml})$. Four groups were studied: (Group 1) 24-hr culture in media followed by 24-hr treatment without inflammatory mediators (CTRL); (Group 2) ethanol pretreatment (24-hr incubation with 100mM ethanol) followed by 24-hr induction with simulation with inflammatory mediators in media in the absence of ethanol (E/M); (Group 3) 24-hr culture in media without ethanol followed by 24-hr with ethanol present along with inflammatory mediators $(\mathrm{M} / \mathrm{E})$, and (Group 4) ethanol present during the pretreatment as well as during treatment with inflammatory mediators (E/E). Production of nitric oxide was measured as the accumulation of nitrite and nitrate, using the Griess assay. In a separate experiment, the cells were treated with $\mathrm{N}^{\mathrm{G}}$-monomethyl-L-arginine (NMA), competitive NOS inhibitor, during stimulation with inflammatory agents. At the end of incubation period, both nitrite and nitrate were measured. This was accomplished by using the Greiss method for colorimetrically determining the amount of nitrite in the medium. A second measurement, after the medium had been treated with $E$. coli nitrate reductase to quantitatively convert nitrate to nitrite, yielded the total nitrite plus nitrate (NOX). The difference between these measurements yielded the amount of nitrate.

\section{Statistical Analysis}

All the results are expressed as means \pm SEM values. The Turkey t-test was applied to data from the FTO2B cells and hepatocytes. Values were considered significant at $\mathrm{P}<0.05$.

\section{Results}

\subsection{NO Formation in Primary Hepatocytes}

The results of this study are shown on Figures 1-5 below. Cells cultured with or without ethanol produced

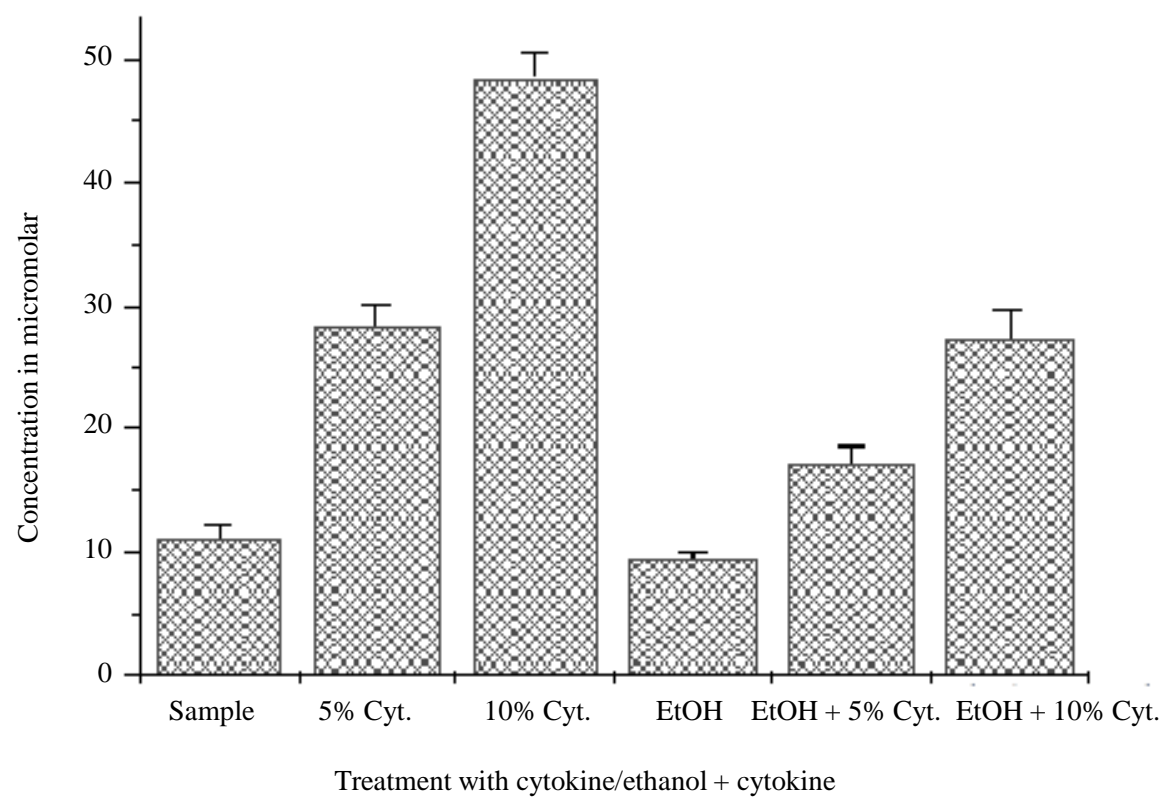

Figure 1. Effect of ethanol on graded doses of inflammatory mixture (5\% to $10 \%$ of cytokine mix + LPS). Nitrite plus nitrate (NOX) produced by hepatocytes. The bars indicate mean \pm SEM values of 7 to 10 experiments. 


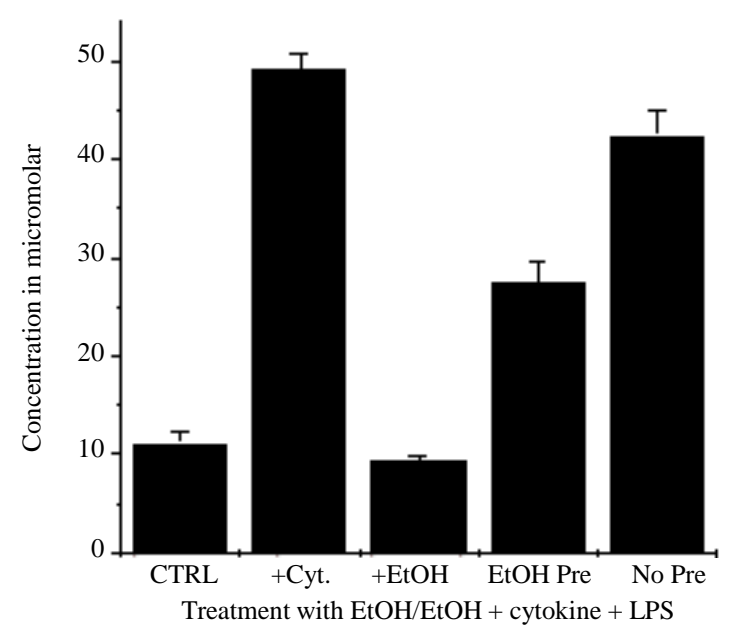

Figure 2. Ethanol decreases nitric oxide production by hepatocytes. CTRL = Hepatocyte control cultured without inflammatory mixture (Cyt.); +Cyt. = 24-hr culture followed by 24-hr stimulation with inflammatory mixture; $+\mathrm{EtOH}=24$-hr culture in $100 \mathrm{mM}$ EtOH alone; EtOH Pre = 24-hr pretreatment with $100 \mathrm{mM}$ EtOH followed by 24-hr stimulation with inflammatory mixture in the presence of EtOH; No Pre = 24-hr culture in media followed by 24-hr stimulation with inflammatory mixture in EtOH.

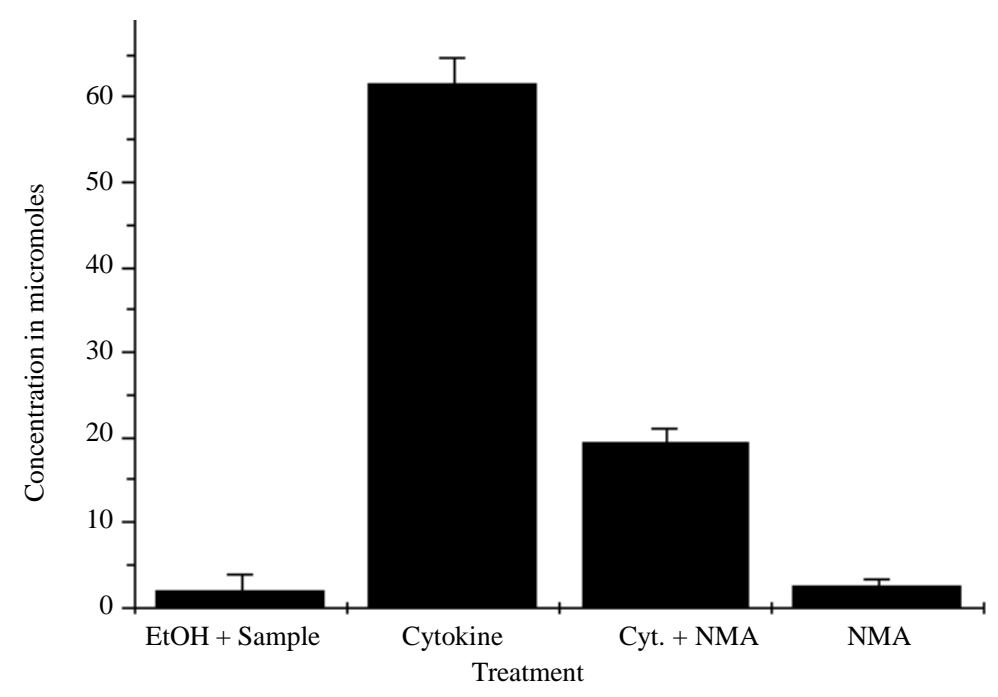

Figure 3. Nitric oxide production by FTO2B cells with or without ethanol. Inhibition of NO production was observed when the hepatocytes were treated with NG-monomethyl-L-arginine (NMA) during stimulation. EtOH + Sample $=\mathrm{EtOH}+$ FTO2B; Cytokine $=$ Cytokine + FTO2B; Cyt. + NMA = Cytokine + NMA; NMA $=$ FTO2B + NMA only.

little or no amount of NO without inflammatory mediators. However, when cells were stimulated with inflammatory mediators, they produced a significant amount of NO (Figure 1). NO production was reduced in hepatocytes pretreated or cotreated with ethanol. However, ethanol inhibition of NO production was more observed when hepatocytes were pretreated and incubated with ethanol (EE) than without pretreatment (ME). Effect of ethanol on NO production was observed with graded doses of inflammatory mixture (Figure 2). Using 10\% of the mixture produced 50\% inhibition of NO production in the presence of ethanol. Inhibition of NO production was observed when hepatocytes were treated with $\mathrm{N}^{\mathrm{G}}$-monomethyl-L-arginine (NMA) during stimulation (Figure 3) indicating the source of NO that was produced. NO formation is inhibited in primary rat hepatocytes with ethanol pretreatment (Figure 4). 


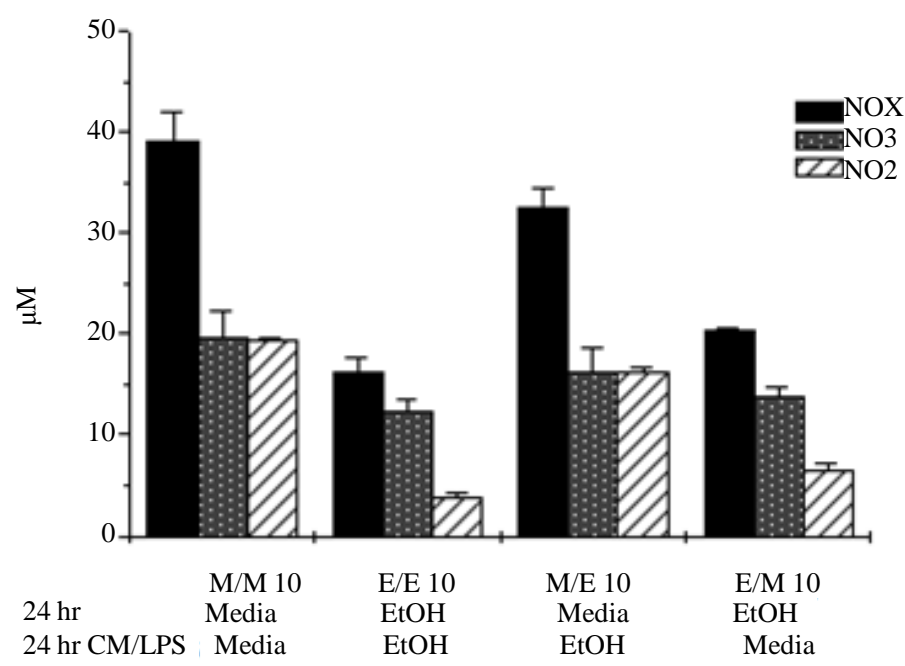

Figure 4. Effect of EtOH pretreatment/co-treatment on NO formation by Cytokine-LPS-stimulated hepatocytes. M/M $10=24$-hr culture in media followed by 24-hr treatment with inflammatory mediators; E/E 10 $=$ ethanol present during the pretreatment as well as during treatment with inflammatory mediators; M/E $10=24$-hr culture in media without ethanol followed by 24-hr with ethanol present along with inflammatory mediators; E/M 10 = ethanol pretreatment (24-hr incubation with $100 \mathrm{mM}$ ethanol) followed by $24-\mathrm{hr}$ induction with simulation with inflammatory mediators in media in the absence of ethanol.

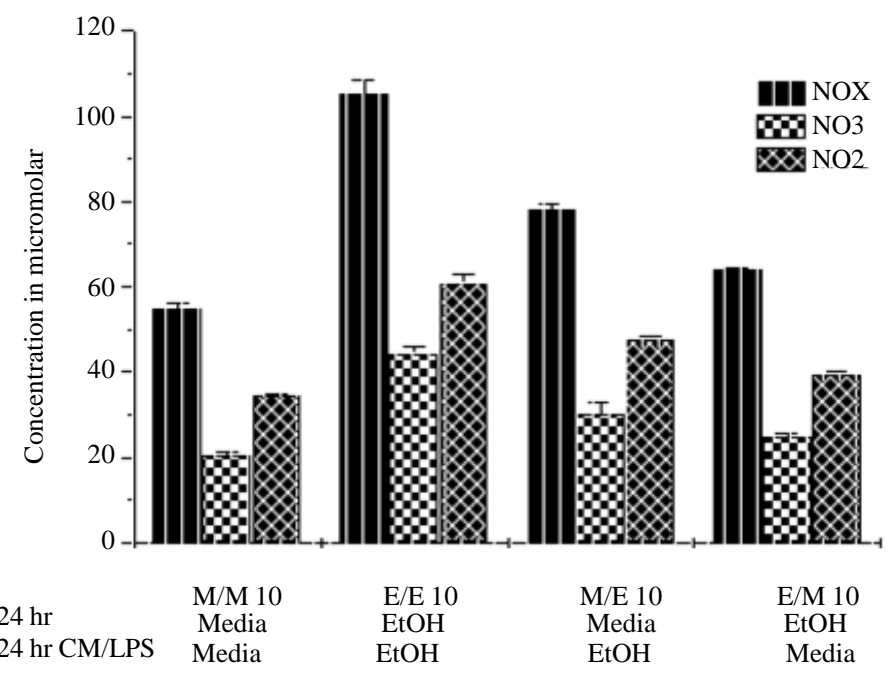

Figure 5. Effect of EtOH pretreatment/co-treatment on NO formation by Cytokine-LPS stimulated FTO2B cells. M/M $10=24$-hr culture in media followed by 24-hr treatment with inflammatory mediators; E/E 10 = ethanol present during the pretreatment as well as during treatment with inflammatory mediators; M/E $10=24-\mathrm{hr}$ culture in media without ethanol followed by 24-hr with ethanol present along with inflammatory mediators; E/M 10 = ethanol pretreatment (24-hr incubation with $10 \mathrm{mM}$ ethanol) followed by $24-\mathrm{hr}$ induction with simulation with inflammatory mediators in media in the absence of ethanol. NOX represents nitrite plus nitrate.

\subsection{NO Formation in FT02B Cells}

Ethanol pretreatment or co-treatment potentiated NO formation in FTO2B cells (Figure 5). Without stimulation 
of FTO2B cells with inflammatory mediators, there was little or no production of NO. In both ethanol treated and nonethanol treated samples, the amount of nitrite produced was more than the nitrate. The highest amount of NO, $105 \mu \mathrm{M}$ was produced when ethanol was used as pretreatment and co-treatment at the same time (E/E), whereas stimulating FTO2B cells without ethanol (M/M) produced $53 \mu \mathrm{M}$ of NO, about $50 \%$ enhancement. Stimulating the cells with inflammatory mixture in the presence of ethanol (without EtOH pretreatment, M/E) yielded $80 \mu \mathrm{M}$ of NO, and stimulating the cells after 24 hrs pretreatment with EtOH (E/M) produced $60 \mu \mathrm{M}$ of $\mathrm{NO}$ indicating that the presence of EtOH at the time of stimulation with inflammatory mixtures enhances more NO production. This disparity was observable when $5 \%$ of inflammatory mix was used.

\section{Discussion}

The immunomodulatory effect of alcohol is known. Some clinical and experimental evidence suggest that alcoholism predisposes to infection and increases its severity [5]. Ethanol effect was observed with EtOH pretreatment where the cells were initially treated with EtOH for 24 hrs and then stimulated with inflammatory mediators in $100 \mathrm{mM} \mathrm{EtOH}$ for another 24 hrs. NO formation was inhibited in primary rat hepatocytes with ethanol pretreatment. Co-treatment with EtOH did not produce significant effect even though diminution in amount of NO produced was observed. This might be suggestive of the fact that deleterious effect of ethanol is more observable during chronic ingestion than when used acutely. Inhibition of NO production was observed when the hepatocytes were treated with $\mathrm{N}^{\mathrm{G}}$-monomethyl-L-arginine (NMA) during stimulation (Figure 2), demonstrating that NO formation was due to specific enzyme NO synthase. However, in FTO2B cells, pretreatment with EtOH consistently enhanced NO production during stimulation. With FTO2B cells, EtOH effect was more pronounced with pretreatment than with co-treatment indicating that ethanol has more effect when used chronically. It was also observed that more nitrite than nitrate was produced in the presence of ethanol. Chronic alcohol abuse is associated with damage to multiple organs, including brain, liver, pancreas, cardiovascular system, skeletal muscles, and the unborn fetus. Evidence suggests that chronic alcohol consumption inhibits some of the protective and proliferative responses that normally occur in hepatocytes especially those that have been exposed to inflammatory agents like TNF- $\alpha$ [2]. These workers demonstrated that chronic, but not acute, ethanol administration increases peroxynitrite hepatotoxicity by enhancing concomitant production of nitric oxide and superoxide. The cumulative effects of these processes cause hepatocyte death while impairing the liver's normally robust regenerative response, and then, serious liver damage. Generation of reactive oxygen species (ROS) is presumed to be the key causative event in oxidative injury. Some investigators also showed that Soymida februfuga ethanol extract can protect against ethanol induced oxidative damage in HepG2 cells [14]. The effect of NO on oxidative stress in the liver appears complex since both pro-oxidant and antioxidant properties have been reported by various investigators. As a pro-oxidant, NO could lead to secondary formation of highly oxidizing molecules. NO and superoxide anion react to form a peroxynitrite anion that can be protonated rapidly to yield hydroxyl radical-like and nitrogen dioxide [3] [9] [15]. These studies indicate that ethanol toxicity is mediated by peroxynitrate and the peroxynitrite-mediated damage to isocitrate dehydrogenase (ICDH) and superoxide dismutase (SOD) may result in perturbation of cellular antioxidant defense systems and subsequently lead to a pro-oxidant condition. Inhibition of NO production has been reported to result in enhanced formation of superoxide anion in the perfused liver [3]. Some investigators have shown that NO plays a double role in the liver: NO biosynthesis in hepatocytes protects against ethanol-induced oxidative stress whereas NO production in macrophages prevents the NO protection of hepatocytes through prostaglandin release [16]. These workers suggested that clinical use of NO donors or NO synthase inhibitors in patients with inflammatory disease and monocyte/macrophage infiltration of liver, such as alcoholic hepatitis, could be ineffective and even harmful. While alcohol may produce deleterious effect in some organs, some beneficial effect has been demonstrated in the heart. Our finding shows that alcohol effect differs in various cells since it decreased NO formation in primary rat hepatocytes and enhanced NO production in rat hepatoma cell line, FTO2B. This indicates that ethanol may produce different effects which may depend on the status of the liver in individuals. Differential effect of ethanol has been demonstrated [17] [18]. These investigators observed that in sinusoidal endothelial cells, LPS tolerance increased the NO production in males and decreased it in the females. Additional ethanol treatment failed to influence NO production in males, but reversed the tolerance effect in female rats. Other investigators have demonstrated modulation of murine serum TNF and IL-6 levels by female sex hormones in endotoxemia [19]. The cell specificity of ethanol may have some implications in the therapy of liver diseases while beneficial effects of NO can be used in the therapy of other diseases [20]. More research remains to be done in this area. 


\section{Conclusion}

In conclusion, ethanol alone does not appear to have a direct effect on nitric oxide synthase. Its effect is secondary since it did not affect NO formation in the absence of stimulation by inflammatory mixture. Our data indicates that NO formation is enhanced in FTO2B cells but inhibited in primary rat hepatocytes with ethanol pretreatment.

\section{References}

[1] Center for Disease Control (CDC) (2014) Alcohol and Public Health: Fact Sheets-Alcohol. http://www.cdc.gov/alcohol/fact-sheets.htm

[2] Cederbaum, A.I. (2012) CYP2E1 Potentiates Toxicity in Obesity and after Chronic Ethanol Treatment. Drug Metabolism and Drug Interaction, 27, 125-144. http://dx.doi.org/10.1515/dmdi-2012-0014

[3] Baraona, E., Zeballos, G.A., Shoichet, L., Mak, K.M. and Lieber, S.S. (2002) Ethanol Consumption Increases Nitric Oxide Production in Rats and Its Peroxynitrite-Mediated Toxicity Is Attenuated by Polyenylphosphatidylcholine. Alcoholism: Clinical and Experimental Research, 26, 883-889. http://dx.doi.org/10.1111/j.1530-0277.2002.tb02618.x

[4] Nanji, A.A. and Hiller-Sturmhöfel, S. (1997) Apoptosis and Necrosis: Two Types of Cell Death in Alcoholic Liver Disease. Alcohol Health and Research World, 21, 325-330.

[5] Adams, A.G. and Jourdan, C. (1984) Infection in Alcoholic. Medical Clinics of North America, 68, 179-200.

[6] Choi, J. (2012) Oxidative Stress, Endogenous Antioxidants, Alcohol, and Hepatitis C: Pathogenic Interactions and Therapeutic Considerations. Free Radical Biology and Medicine, 52, 1135-1150. http://dx.doi.org/10.1016/j.freeradbiomed.2012.01.008

[7] Cederbaum, A.I. (2001) Introduction-Serial Review: Alcohol, Oxidative Stress and Cell Injury. Free Radical Biology and Medicine, 31, 1524-1526. http://dx.doi.org/10.1016/S0891-5849(01)00741-9

[8] Joshi, M.S., Ponthier, J.L. and Lancaster Jr., J.R. (1999) Cellular Antioxidant and Pro-Oxidant Actions of Nitric Oxide. Free Radical Biology and Medicine, 27, 1357-1366. http://dx.doi.org/10.1016/S0891-5849(99)00179-3

[9] Cederbaum, A.I. (2014) Cyrochrome P450 2E1, Oxidative Stress, JNK, and Autophagy in Acute Alcohol-Induced Fatty Liver. In: Hayat, M. A., Ed., Autophagy: Cancer, Other Pathologies, Inflammation, Immunity, Infection, and Aging, Volume 2: Role in General Diseases, Academic Press, Waltham, 341-356.

[10] Wu, D. and Cederbaum, A.I. (2003) Alcohol, Oxidative Stress, and Free Radical Damage. Alcohol Research and Health, 27, 277-284. http://pubs.niaaa.nih.gov

[11] Zhang, H. and Potter, B.J. (1992) The Effect of Ethanol Metabolism on Ferritin Uptake by Freshly Isolated Rat Hepatocytes: Is Acetaldehyde Responsible for This Alteration? Alcoholism: Clinical and Experimental Research, 16, 301-307.

[12] Potter, B.J., Blades, B., McHUgh, T.A., Nunes, R.M., Beloqui, O., Slott, P.A. and Rand, J.H. (1989) Effects of Endotoxin on Iron Uptake by the Hepatocyte. American Journal of Physiology, 257, G524-G531.

[13] Kim, Y.M., Bergonia, H. and Lancaster Jr., J.R. (1995) Nitrogen Oxide-Induced Autoprotection in Isolated Rat Hepatocytes. FEBS Letters, 374, 228-232. http://dx.doi.org/10.1016/0014-5793(95)01115-U

[14] Jin, M., Ande, A., Kumar, A. and Kumar, S. (2013) Regulation of Cytochrome P450 2E1 Expression by Ethanol: Role of Oxidative Stress-Mediated PKC/JNK/SP1 Pathway. Cell Death and Disease, 4, e554.

[15] Yang, S.U., Lee, J.H. and Park, J. (2008) Ethanol Induces Peroxynitrite-Mediated Toxicity through Inactivation of NADP $^{+}$-Dependent Isocitrate Dehydrogenase and Superoxide Dismutase. Biochimie, 90, 1316-1324. http://dx.doi.org/10.1016/j.biochi.2008.03.001

[16] Griffon, B., Chevanne, M., Morel, P., Cillard, P. and Sergent, O. (2000) Activated Macrophages Increase the Susceptibility of Rat Hepatocytes to Ethanol-Induced Oxidative Stress: Conflicting Effects of Nitric Oxide. Alcohol and Alcoholism, 35, 230-235. http://dx.doi.org/10.1093/alcalc/35.3.230

[17] Wang, E., Spitzer, J.J. and Chamulirat, W. (1999) Differential Regulation of Inducible Nitric Oxide Synthase Gene Expression by Ethanol in the Human Intestinal Epithelial Cell Line DLD-1. Nitric Oxide: Biology and Chemistry, 3, 244-253. http://dx.doi.org/10.1006/niox.1999.0230

[18] Spitzer, J.A. and Spitzer, J.J. (2000) Lipopolysaccharide Tolerance and Ethanol Modulate Hepatic Nitric Oxide Production in Gender-Dependent Manner. Alcohol, 21, 27-35.

[19] Zuckerman, S.H., Bryan-Poole, N., Evans, G.F., Short, L. and Glasebrook, A.L. (1995) In Vivo Modulation of Murine Serum Tumour Necrosis Factor and Interleukin-6 Levels during Endotoxemia by Oestrogen Agonists and Antagonists. Immunology, 86, 18-24.

[20] Moezi, L., Heidari, R., Amirghofran, Z., Nekooeian, A.A., Monabati, A. and Dehpour, A.R. (2013) Enhanced AntiUlcer Effect of Pioglitazone on Gastric Ulcers in Cirrhotic Rats: The Role of Nitric Oxide and IL-1 $\beta$. Pharmacological Reports, 651, 134-143. 
Scientific Research Publishing (SCIRP) is one of the largest Open Access journal publishers. It is currently publishing more than 200 open access, online, peer-reviewed journals covering a wide range of academic disciplines. SCIRP serves the worldwide academic communities and contributes to the progress and application of science with its publication.

Other selected journals from SCIRP are listed as below. Submit your manuscript to us via either submit@scirp.org or Online Submission Portal.
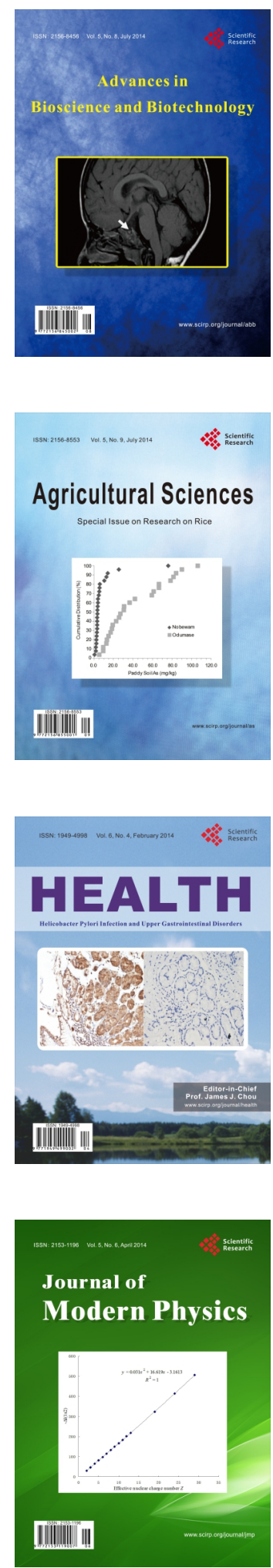
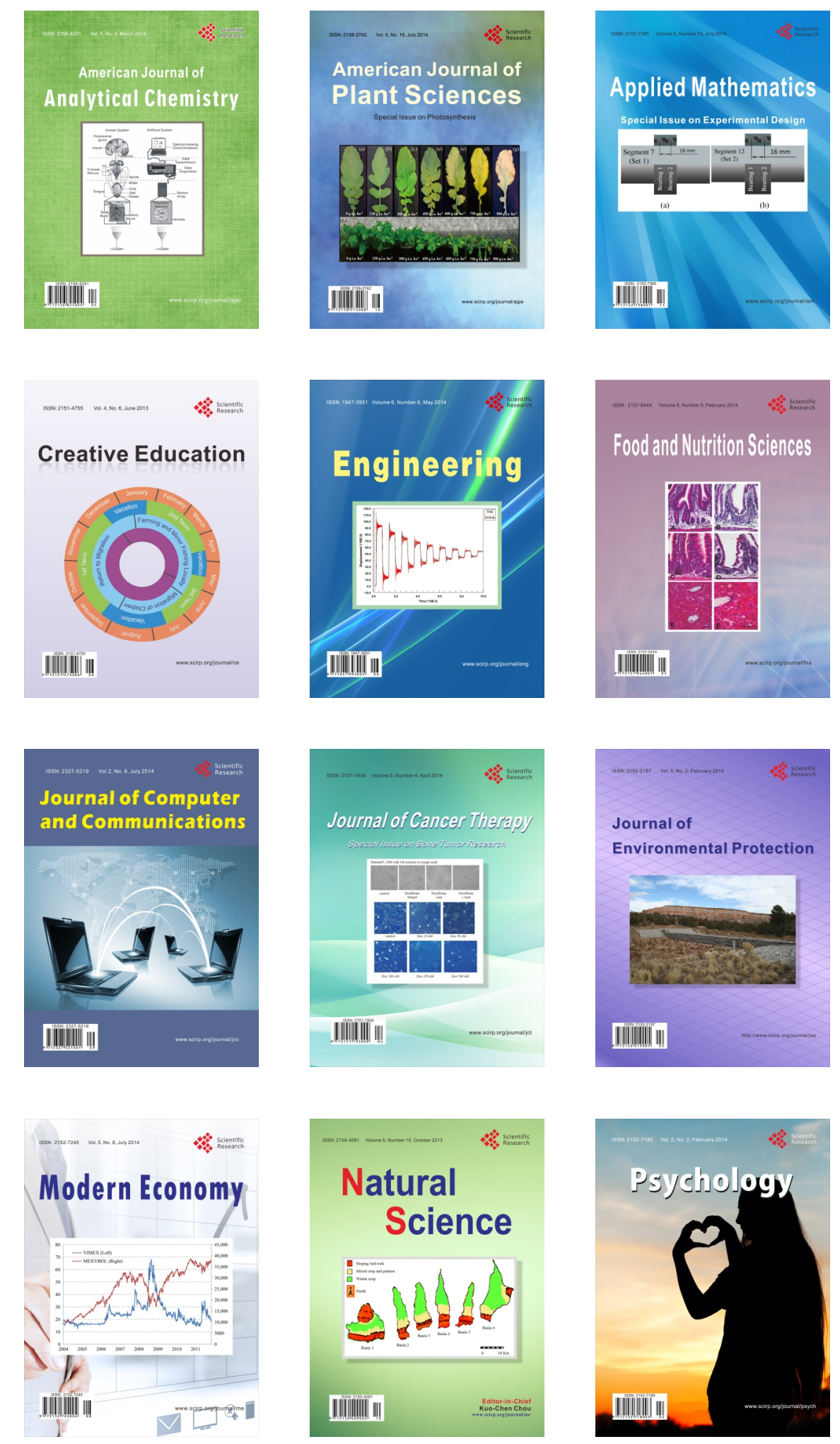